\title{
Review Article ZHENG-Omics Application in ZHENG Classification and Treatment: Chinese Personalized Medicine
}

\author{
Jianye Dai, ${ }^{1}$ Junwei Fang, ${ }^{1}$ Shujun Sun, ${ }^{1}$ Qiwen Chen, ${ }^{2}$ Huijuan Cao, ${ }^{1}$ Ningning Zheng, \\ Yongyu Zhang, ${ }^{1}$ and Aiping $\mathrm{Lu}^{3}$ \\ ${ }^{1}$ Center for Traditional Chinese Medicine and Systems Biology, Shanghai University of Traditional Chinese Medicine, \\ Shanghai 201203, China \\ ${ }^{2}$ Department of Integrated Oncology, Shanghai Cancer Center, Fudan University, Shanghai 200032, China \\ ${ }^{3}$ School of Chinese Medicine, Hong Kong Baptist University, Hong Kong \\ Correspondence should be addressed to Yongyu Zhang; dryyz@sina.com and Aiping Lu; lap64067611@126.com
}

Received 10 January 2013; Revised 22 February 2013; Accepted 27 February 2013

Academic Editor: Zhaoxiang Bian

Copyright (C) 2013 Jianye Dai et al. This is an open access article distributed under the Creative Commons Attribution License, which permits unrestricted use, distribution, and reproduction in any medium, provided the original work is properly cited.

With the hope to provide an effective approach for personalized diagnosis and treatment clinically, traditional chinese medicine (TCM) is being paid increasing attention as a complementary and alternative medicine. It performs treatment based on ZHENG (TCM syndrome) classification, which could be identified clinical special phenotypes by symptoms and signs of patients even if they have a different disease. However, it caused controversy because ZHENG classification only depends on observation, knowledge, and clinical experience of TCM practitioners, which lacks objectivity and repeatability. Although researchers and scientists of TCM have done some work with a lot of beneficial methods, the results could not reach satisfactory with the shortcomings of generalizing the entire state of the body or ignoring the patients' feelings. By total summary, mining, and integration of existing researches, the present paper attempts to introduce a novel macro-microconcept of ZHENG-omics, with the prospect of bright future in providing an objective and repeatable approach for Chinese personalized medicine in an effective way. In this paper, we give the brief introduction and preliminary validation, and discuss strategies and system-oriented technologies for achieving this goal.

\section{Introduction}

After the accomplishment of the Human Genome Project, personalized medicine is looming in the horizon. Recognizing continuous genetic variation and expensive cost, it seems that it would be difficult to perform personalized drug therapy for a wide range of major diseases using genomic knowledge alone, although it is clearly important [1]. Alternatively, a holistic approach attempting to bring the body, mind, and spirit into harmony $[2,3]$, traditional chinese medicine (TCM) combined with the principle of "ZHENG (TCM Syndrome) Classification and Treatment" (ZCT, bian zheng lun $z h i$, in Chinese), may bring personalized medicine to light in an efficient way. Researches have been conducted by He et al. [4] and Lu et al. [5] to illustrate that the effective rate of treatment based on ZHENG classification may improve the specificity and efficiency in both TCM and Western medicine.
The success of personalized medicine relies on having accurate diagnostic tests that identify patients who can benefit from targeted therapies [6]. While in TCM, ZHENG classification is performed by four diagnostic methods: looking, listening and smelling, asking, and touching. Still it is argued, as it depended on clinical observation and TCM practitioner's experience, which would be subjective and unrepeatable. So, a great breakthrough in TCM diagnosis with objectivity, repeatability, and comprehensiveness is needed.

Actually, researchers and scientists of TCM have done some work with a lot of beneficial methods, such as physiology and biochemistry [7], molecular biology [8], and tongue image digitization $[9,10]$, to classify ZHENGs and evaluate the treatment based on ZHENG classification. Though the researches are beneficial, they are far from satisfactory. The main reason accounting for the results may be that these methods only focus on one or several indicators, which 
cannot generalize the entire state of the body. However, with the advent of the era of systems biology a broader technology platform was established for the study of TCM. The omic technologies provide integral, systemic, and dynamic technology platforms and lay the foundation for the systemic study at a higher level. The system point of view provides us with an important idea to sweep aside the fog of mysterious TCM and may be a breakthrough point of incorporating Eastern medicine with Western medicine. Though the researches based on system biology have had great progresses recently, there is a little flaw of ignoring patients' feelings in the only omic data.

So, through existing researches of system biology application in ZCT, we would like to propose further a new macromicroconcept, namely, "ZHENG-Omics," which is defined as "a systematic approach for targeting individual patient, guiding treatment, and predicting the outcome of personalized treatment by global NET-Markers (combination of genes, proteins, metabolites, symptoms and others based on the mathematical model), on the basis of ZCT". In this paper, after comprehensive reviewed recent researches, we will gradually show you brief introduction and preliminary validation and discuss strategies and system-oriented technologies for achieving the goal of providing an objective and repeatable approach for Chinese personalized medicine in an effective way.

\section{Research Status of System Biology Application in ZCT}

TCM researchers have attempted some beneficial works. Research in the area of Yin-Yang sets a good example. YinYang (two opposite, complementary, interdependent, and exchangeable aspects of nature), the general principles in eight, are used to categorize natural phenomena, which helped us understand, prevent, and cure disease. The classification of Yin-Yang usually is the first step in the ZHENG classification. Systems biology was proved to be a useful tool for the study of Yin-Yang, although the concept of Yin-Yang is abstract, mysterious, and obscure. So far the most researches of YinYang syndrome are concentrated on Yin-deficiency syndrome and Yang-deficiency syndrome. Genomic information showed that Yang-deficiency patients had significant difference in Haplotype 25 (Hap25) of APM1 rs7627128, rs1063539, PSMB7, and CXCR4 compared to control group; however, Haplotype13 of PPARG in Yin-deficiency patients [11, 12]. At the proteomic level, it was found that CRP, CRH, IL10, ACE, PTH, MPO, CRH, PTH, PRL, BRCA1, BRCA2 [13], transthyretin, plasma retinol-binding protein precursor and chain A-prealbumin [14], liver protein, and protein of the liver mitochondria [15] levels correlated with Yang-deficiency syndrome. It is especially worth noting that metabonomics is widely and deeply utilized in Yin-Yang classification. Wang et al. [16] and Lu et al. [17] have, respectively, found different metabolites between Kidney-Yin and Kidney-Yang deficiency syndrome, which are estrone, creatinine, uric acid, indoxyl sulfate and so on, demonstrating the notable differences of these two ZHENGs. From these previous researches we realized that it is possible to establish a group of preliminary global net-markers to identify the Yin-Yang deficiency syndrome. Though there has been no research on NetMarkers of Yin-Yang syndrome, the combination of network biology and Bioinformatics has been used by Li et al. to investigate novel biomarkers for cold-heat syndrome which partly represents the Yin-Yang syndrome in TCM $[18,19]$.

However, ZHENG classification is not the ultimate goal, but a progression towards treatment and curing of diseases. Unfortunately, current evaluation methods are conducted ignoring theories of TCM and are unable to bring this traditional medicine which has been validated in clinical practice to light. This may cripple public confidence in the effectiveness of TCM. To counter this, an objective method on the basis of holistic TCM must be developed. What is gratifying systems biology, especially metabonomics, has shown promising application prospect in its practice. For example, it was demonstrated in our previous experiment that if different ZHENGs of hepatitis-B-caused cirrhosis patients were treated by the same therapy, they would show various responses [20]. Similar research has been performed by Chen et al. [21] to study the biochemical profiles of hydrocortisoneinduced animal models, which evaluated the effectiveness of Herba Cistanches Deserticolae that is formulated to warm and reinforce kidney Yang to intervene Kidney-Yang deficiency rats. A good correlation between the chemical profile and the progress of treatment was observed. In addition to this study, Wang et al. [16] and Lu et al. [17] evaluated the effectiveness of Liuwei Dihuang Pill and Rhizoma Drynariae, respectively, in Kidney-Yin and Kidney-Yang syndromes and satisfactory results were obtained as well.

Due to space limitation and insufficient research, other research has not been touched upon. To systematically note ZHENG research as being performed presently, clinical and experimental studies have been summarized in Tables 1 and 2 , with respect to the combination of ZHENG in TCM and disease in western medicine.

\section{Brief Introduction of the Origin of "ZHENG-Omics"}

Our thought is not groundless but derived from former researches, although most of which were discrete and without a systemic and consistent research strategy. ZHENG-Omics is not simple combination of systems biology and ZCT. Their association could play bigger roles in helping mutually to remedy deficiencies. One, the systems biology research and philosophy of TCM coincide in many ways. They share similar attributes in many aspects, especially in a holistic approach. Two, the strategy employing a dynamic noninvasive approach is very important for the acquisition of longterm, large-scale samples. The thorough knowledge of ZHENGs' connotation and intervention may evolve to a dynamic classification approach. Three, a nontargeting concept may provide freedom for exploration of unknown classifications of disease states without being bound by the existing methods familiar to Western medicine. Finally, digital results can be integrated for ZHENG classification through 
TABLE 1: Clinical researches of TCM ZHENGs with systems biology.

\begin{tabular}{|c|c|c|c|}
\hline Syndromes & Disease & Researchers & Omics \\
\hline \multirow{2}{*}{ Liver-gallbladder dampness-heat } & Hypertension & Chu et al. [22] & Proteomics \\
\hline & Chronic hepatitis B & Guo et al. [23] & \\
\hline Liver Yang transforming into wind & Cerebral infarction & Zeng et al. [24] & Proteomics \\
\hline Liver stagnation & $\begin{array}{c}\text { Depression, premenstrual syndrome, } \\
\text { menopausal syndrome }\end{array}$ & Tan et al. [25] & Proteomics \\
\hline \multirow{3}{*}{ Cold and heat pattern } & Rheumatoid arthritis & Lu et al. [26] & Genomics \\
\hline & Cold-syndrome genealogy & Wang et al. [27] & Genomics \\
\hline & Rheumatoid arthritis & Gu et al. [28] & Metabolomics \\
\hline \multirow{3}{*}{ Spleen deficiency } & Colorectal cancer & Yang et al. [29] & Genomics \\
\hline & Chronic superficial gastritis & Yang et al. [30] & Genomics \\
\hline & Gastritis & Liu et al. [31] & Proteomics \\
\hline \multirow{5}{*}{ Kidney deficiency } & Diabetes & Weng et al. [32] & Genomics \\
\hline & & Wu et al. [11] & Genomics \\
\hline & Normal & Ni et al. [12] & Genomics \\
\hline & Normal & Liu et al. [14] & Proteomics \\
\hline & Chronic heart failure & Zheng et al. [33] & Metabolomics \\
\hline \multirow{2}{*}{ Dampness syndrome } & Chronic hepatitis B & Guan et al. [34] & Genomics \\
\hline & Chronic gastritis & Wang et al. [35] & Proteomics \\
\hline Dampness-phlegm & Obesity & Wang et al. [36] & Genomics \\
\hline \multirow{5}{*}{ Blood stasis } & Coronary heart disease & Ma et al. [37] & Genomics \\
\hline & Coronary heart disease & Yuan et al. [38] & Genomics \\
\hline & Coronary heart disease & Yuan et al. [39] & Genomics \\
\hline & Coronary heart disease & Wu et al. [40] & Proteomics \\
\hline & Unstable angina & Wang et al. [41] & Metabolomics \\
\hline \multirow{5}{*}{ Deficiency syndrome } & Hepatitis B cirrhosis & Li et al. [42] & Genomics \\
\hline & Chronic hepatitis B & Song et al. [43] & Proteomics \\
\hline & Primary liver cancer & Chen et al. [44] & Metabolomics \\
\hline & Diabetes mellitus & Wu et al. [45] & Metabolomics \\
\hline & Hypertension & Yang et al. [46] & Metabolomics \\
\hline Liver-kidney Yin deficiency syndrome & Hepatocellular carcinoma & Weng et al. [47] & Genomics \\
\hline Ascendant hyperactivity of Ganyang syndrome & Hypertension & Jiang et al. [48] & Metabolomics \\
\hline
\end{tabular}

statistical analysis and database building and modeling. Most importantly, digitalization of information can be absorbed from scholars without a TCM background into this field of research.

The scope of ZHENG-omics can be illustrated as follows. Firstly, clinical symptoms are classified into several groups according to the principle of ZHENG classification and the difference in biological markers, including DNA, RNA, proteins, and metabolites, are identified among these groups. Secondly, by integrating of genes, proteins, metabolites, symptoms, and others, NET-Markers of ZHENG will be obtained from former differences by bioinformatics and other mathematical analysis. The markers could then be used to provide the basis in developing a possible population-screening tool for selecting target individuals and creating evaluation index for personalized treatment based on ZHENG classification. Finally, ZHENG-Omics will give an objective and practical evaluation to the classical ZCT.

\section{Preliminary Validation}

To explore the feasibility of our thought process, a preliminary case study was performed. The study was performed in accordance with the principles contained in the Declaration of Helsinki and was approved by the local ethics committee. Urine samples from 12 healthy volunteers (control group, CG) and 17 patients (Hepatitis-B-caused cirrhosis group, HBCG) were analyzed by gas chromatography mass spectrometry (GC/MS), and multivariate statistical analysis was performed by Simca-P 12.0 Software package (Umetrics, Umea, Sweden) [68]. Though the patients were treated by Fuzheng Huayu tablets (FZHY tablets, Chinese patent medicine) in the same way as lacking significant deviation in Child-Pugh Score, they were classified into 2 ZHENG types by the TCM practitioner according to the recording symptoms: liver-gallbladder dampness-heat syndrome (LGDHS, $n=7$ ) and liver-kidney Yin deficiency syndrome (LKYDS, $n=10$ ) [69]. The TCM 
TABLE 2: Experimental researches of TCM ZHENGs with systems biology.

\begin{tabular}{lccc}
\hline Syndromes & Disease & Researchers & Omics \\
\hline & Migraine headache & Hu et al. [49] & Proteomics \\
Ascendant hyperactivity of liver Yang & Migraine headache & Zhong et al. [50] & Proteomics \\
& Hypertension & Zhou et al. [51] & Proteomics \\
& Hypertension & Zhang et al. [52] & Proteomics \\
\hline Liver depression & & Zhong et al. [53] & Proteomics \\
\hline Spleen deficiency & & Wang et al. [54] & Proteomics \\
& & Luo et al. [55] & Getabolomics \\
\hline & Chronic heart failure & Tang et al. [15] & Proteomics \\
Kidney deficiency & & Zheng et al. [33] & Metabolomics \\
& & Lu et al. [17] & Metabolomics \\
\hline Blood stasis-phlegm & Wang et al. [16] & Metabolomics \\
\hline Blood deficiency & Chen et al. [57] & Metabonomics \\
\hline Blood stasis & Coronary heart disease & Liu et al. [58] & Proteomics \\
\hline Yang Qi deficiency & Coronary heart disease & Jian et al. [60] & Genomics \\
\hline
\end{tabular}

ZHENG types were identified by three chief or deputy physicians according to "evaluation criteria of the clinical diagnosis, drug efficacy and ZHENG classification for cirrhosis (pilot program)" [70]. This classification was then verified by metabonomics. (Figure 1).

Furthermore, there was a significant difference in metabolites between CG and two ZHENGs which were selected by OPLS loading plot analysis. The differentiated metabolites combined with classic symptoms and feelings (bitter taste, hypochondriac pain, slimy fur of tongue, and yellow fur of tongue in LGDHS, while dry mouth, lack of strength, without fur of tongue, and red tongue in LKYDS) were chosen as the potential markers for each ZHENG. The reversions of these metabolites and hierarchical corresponding symptoms were used as indicators of the therapeutic effect of FZHY tablets and found that FZHY tablets are more effective for LKYDS than for LGDHS, which coincides with the treatment principles of TCM (Figure 2). This section relates to the FZHY study only. Further details are provided in the Supplementary Methods available online at http://dx.doi.org/ $10.1155 / 2013 / 235969$.

Because every “-Omics” has the similar features in Systems Biology, as holistic aspects, noninvasive, integrity, multitarget, high-throughput and digitalization, the similar thought could be applied to other "-Omics." It is to say that gene sequences or proteins could be combined with clinical information to provide the NET-Marker of the special ZHENG. Thus, our initial exploration provided strong evidence of the feasibility and robustness of ZHENG-Omics, even though we did not obtain a full validation.

\section{Discussion and Prospect}

The outcome of our exploration is inspiriting us to assess the curative effect based on ZCT by ZHENG-omics that

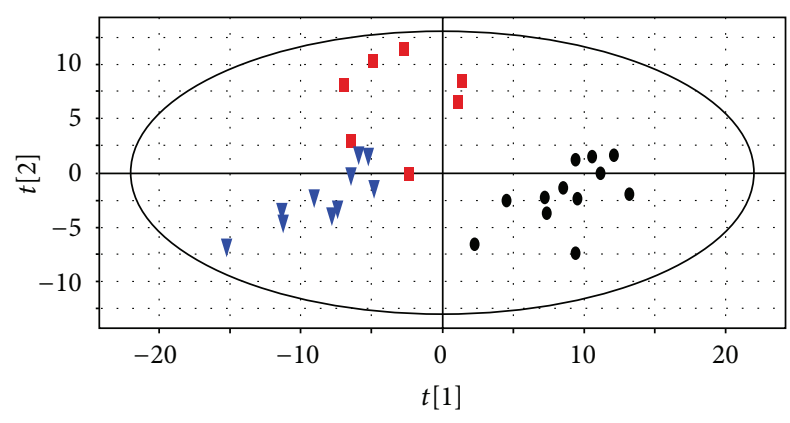

FIGURE 1: PLS-DA score plots between healthy subjects and Hepatitis B caused Cirrhosis subjects. Black dots, Red boxes, blue triangles refer to healthy subjects, Liver-Gallbladder Dampness-Heat Syndrome and Liver-Kidney Yin Deficiency Syndrome of Hepatitis B caused Cirrhosis subjects, respectively.

combines omic data with clinical symptoms and feelings. We hope this approach will narrow the gap between mainstream medicine and TCM.

Compared with pharmaco-omics [1] and other "-Omics," ZHENG-omics may show some promise as a new method having distinct advantages. First of all, ZHENG-omics may give the guide to pharmaco-omic performance with the clinical experience of 3000 years based on ZCT, though lacking enough evident ascertainments. The ZHENG classification may guide patient classification of predose phenotype and prescribe drugs according to the phenotype. Furthermore, ZHENG-Omics could provide the profound connotation of the disease, which consists of not only genetic and environmental factors but also emotional and spiritual factors. This notion resembles the new treatment concept of modern medicine that a treatment should not only relieve symptoms but should also take care of the mental health of a patient. In 


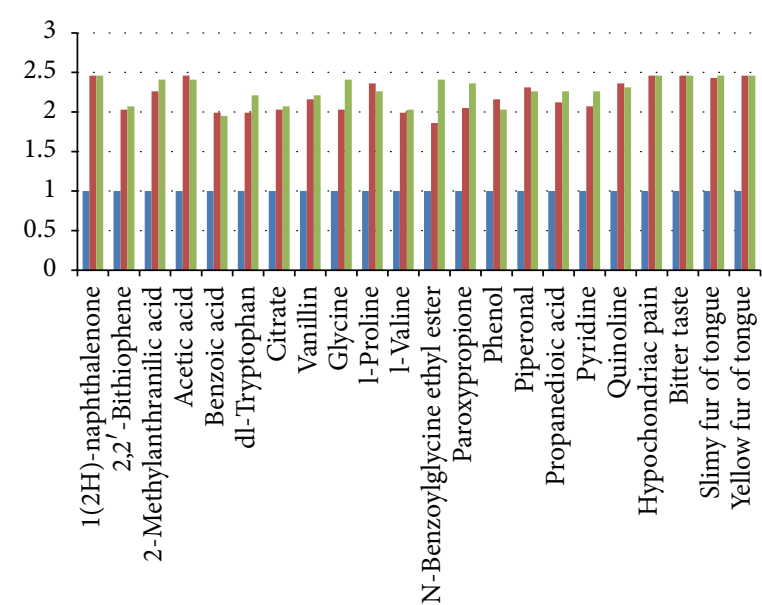

(a)

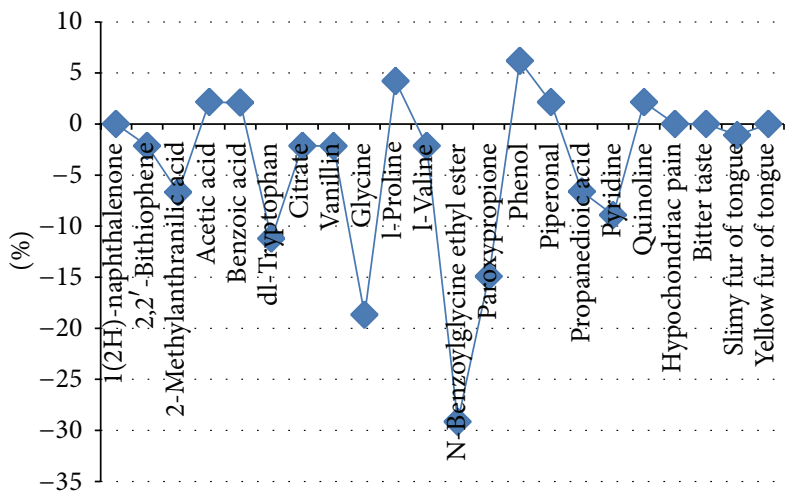

(c)

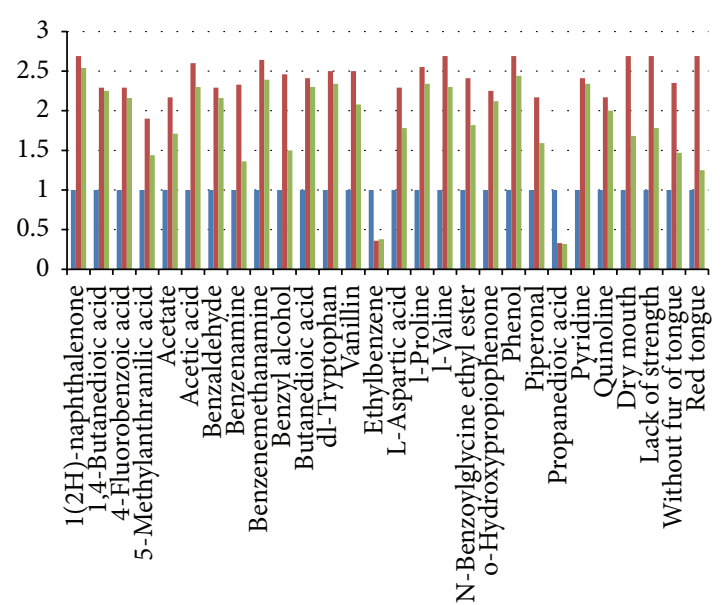

(b)

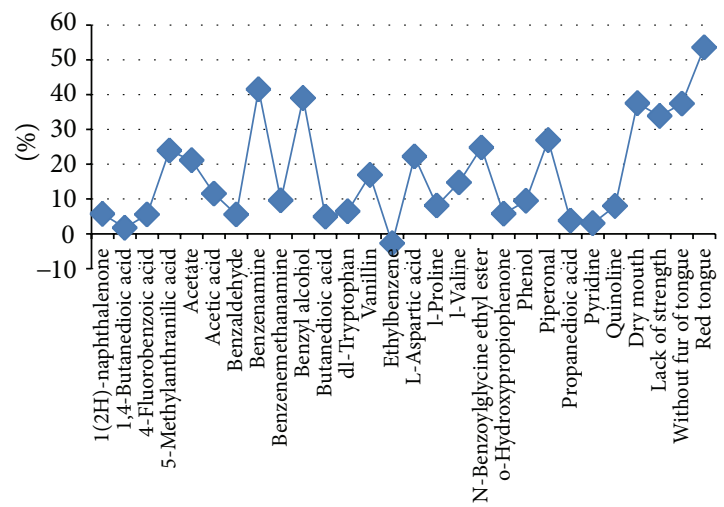

(d)

FIGURE 2: The reversion maps reveal the therapeutic effects of two TCM ZHENG types in hepatitis-B-caused cirrhosis. (a) and (b) Histogram of the therapeutic effects of FZHY Tablet for liver-gallbladder dampness-heat syndrome and liver-kidney Yin deficiency syndrome, respectively, by the changing trend of significantly differential metabolites and classic symptoms. Blue, red, and green bars stand for control group, before intervention and 12-week intervention of FZHY tablet. The $x$-axis represents the changed metabolites and symptoms, and the $y$-axis is fold change of mean ranks calculated by the Mann-Whitney test, compared with the control group. (c) and (d) Curve diagram of regression trend of significantly differential metabolites and classic symptoms in liver-gallbladder dampness-heat syndrome and liver-kidney Yin deficiency syndrome respectively, for 12-week intervention of FZHY tablet. The $x$-axis represents the changed metabolites and symptoms, and the $y$-axis is the rate of reversion, representing the therapeutic effect of FZHY tablet for different ZHENGs.

this respect, ZHENG-omics may provide some new revelations for personalized medicine.

However, every coin has two sides, so as to current "Omics." Every "Omic" has the advantages and disadvantages (Table 3). If using only one "Omics" for study of ZHENG, the shortcomings such as deficiency of cross-reference and scientific discrimination, overloading information, and excessive simplification will show up. On the other side, one can carry out ZHENG-omics at different levels in multiple research centers. Such research can find those specific and meaningful markers distinguishing primary factors from secondary factors. With the multidimensional studies of DNA, proteins, and metabolites, the variability, integrity, and complexity of TCM ZHENGs will be annotated commendably and can be used as basis for understanding and differentiating them. Furthermore, we should combine microscopic omic data with macroscopic symptoms, with the methods such as association rules [71] and bioinformatics [72]. Yet, for ZHENG-omics study, one should pay special attention to the following aspects.

First of all, careful screening and data collection of samples from patients with the most classic ZHENGs are necessary. Before this can be initialized, the patients with classic ZHENG should be targeted, according to the most typical syndromes getting out from literature retrieval and data mining of a lot of clinical cases $[73,74]$. Patient samples for a special ZHENG can be found based on these typical symptoms. The more samples that are collected, the more precision will be developed to the essence of $\mathrm{ZHENG}$, leading to more accurate and objective classification.

Second, the existing strategy should be refined. Since one ZHENG (one syndrome) can change to another ZHENG 


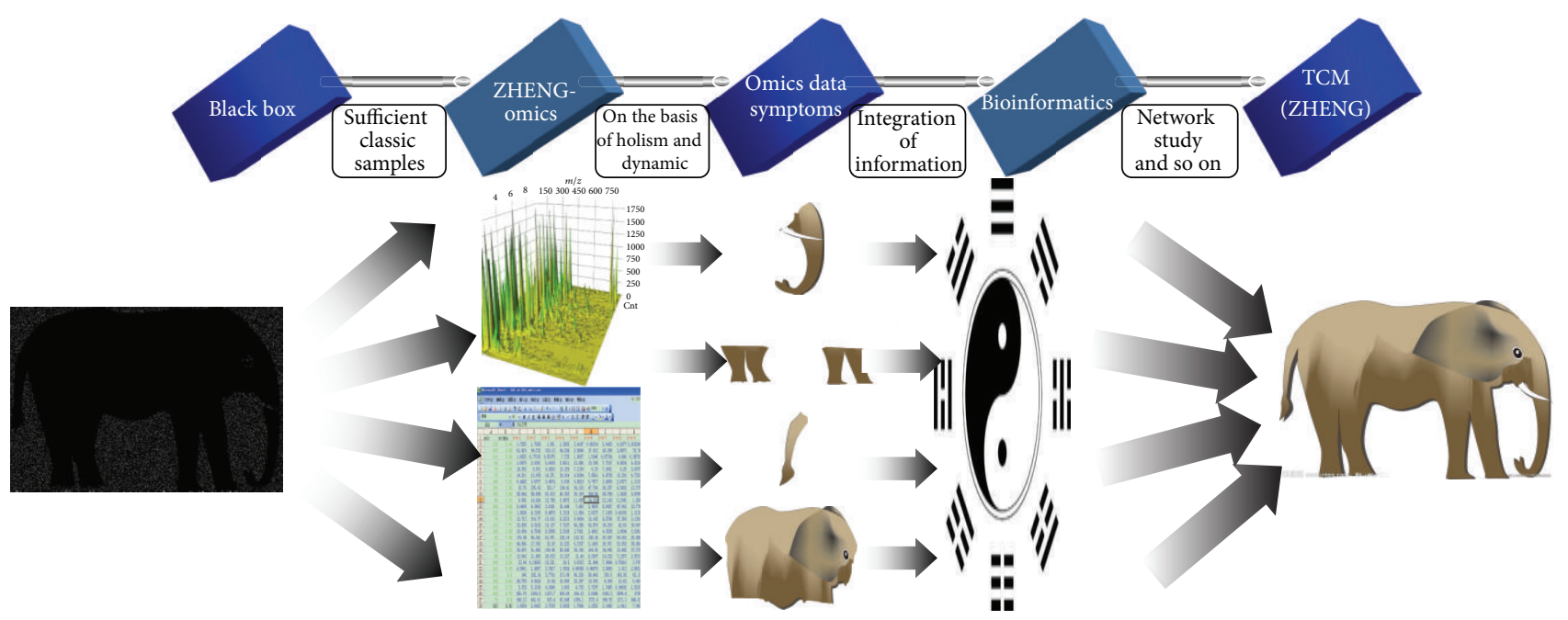

FIGURE 3: Schematic diagram of research approach for ZHENG-omics. Traditional Chinese medicine is mysterious and obscure, like a fuzzy picture. It is difficult to obtain systematic knowledge from the whole directly, yet the partial information is much easier to be acquired and understood, such as nucleic acids, proteins, and metabolites. Combined with classic symptoms and feelings, the former could be obtained by ZHENG-omics from sufficient classic samples on the basis of holism. The integration of omic data and symptoms will be performed by bioinformatics and so on, which further provide the multilevel information, like small lights in the big black box. Then, a vivid picture may emerge in our sights, just like getting the systematic connotation of TCM or ZHENG.

TABLE 3: Brief introduction of “-omics" and bioinformatics.

\begin{tabular}{|c|c|c|c|}
\hline Omics & Advantages & Disadvantages & Literatures \\
\hline $\begin{array}{l}\text { Genomics } \\
\text { (transcriptomics) }\end{array}$ & $\begin{array}{l}\text { Gene polymorphism } \\
\text { Susceptibility for prognosis and treatment } \\
\text { Completed database } \\
\text { High throughput }\end{array}$ & $\begin{array}{l}\text { Nonassociation to regulation of } \\
\text { life activities } \\
\text { Nonconsistent strictly with } \\
\text { mRNA expression }\end{array}$ & $\begin{array}{l}\text { Wu et al. [11] } \\
\text { Lu et al. [63] }\end{array}$ \\
\hline Proteomics & Performer of life function & $\begin{array}{l}\text { Instability } \\
\text { Variability }\end{array}$ & $\begin{array}{l}\text { Liu et al. [64] } \\
\text { Lu et al. [65] }\end{array}$ \\
\hline Metabonomics & $\begin{array}{l}\text { Amplified action } \\
\text { Simplicity to detect } \\
\text { Less numbers } \\
\text { Similarities in different species }\end{array}$ & $\begin{array}{l}\text { Lack of beneficial supports } \\
\text { Interferences by physiological } \\
\text { factors }\end{array}$ & $\begin{array}{l}\text { van Wietmarschen et al. [66] } \\
\text { Sun et al. [67] } \\
\text { Liu et al. [31] }\end{array}$ \\
\hline Bioinformatics & $\begin{array}{l}\text { Totally holism } \\
\text { Exploration the potential of information, } \\
\text { Focusing on function relation }\end{array}$ & Needing of self-development & Li et al. [18] \\
\hline
\end{tabular}

dynamically, the current static study cannot meet the needs of deep research and clinical application. Luckily, ZHENGomics provides a high-throughput and noninvasive clinical research tool and enables study on the dynamical ZHENG classification in an extending period. Particularly, we advocate that initiative and passive intervention could be used to study development and dynamic Classification of ZHNEG, with the help of tracer techniques such as fluorescence labeling. Nowadays, most attention is paid to preliminary intervention by inducers $[1,21]$ and environmental factors [75], while purposeful, further continuous intervention will provide deeper vision into the regularity of ZHENG classification.

Since ZHENGs are frequently considered as phenotypes of a disease, researchers are mostly focused on the different ZHENGs of the same disease. However, we should pay more attention to the formerly mentioned phenomenon that the same syndrome appears in different diseases. This part will bring another look to old drugs as treatments for other diseases.

\section{Conclusion}

Just like a blindfold boy will feel an elephant, snake, tree, wall, and rope would be in his mind when he touched the squirming trunk, grossus knee, broad body, and swinging tail, respectively. He may perceive the whole elephant from combination of these incomplete parts. Huge and metaphysical TCM, especially ZHENG, is corresponding to the elephant, while ZHENG-omics will provide the multilevel information, just like omic data and symptoms compared to tusk, knee, and tail. It is important to note that dynamic and intervention will also paint a vivid picture (Figure 3). Under the strategy of 
"black box-partial system-whole system," the connotation of systematic TCM will emerge in our sights.

Furthermore, the ZHENG-omics possesses and integrates the characteristics of both ZHENG and systems biology and may advance TCM to a new level. It would overcome the shortcomings of current methods for evaluating personal diagnosis, complex intervention, and patients' feelings. Through ZHENG-omic study, personal medicine in TCM may emerge eventually and may change the current medical system. We believe that the advantage of TCM in theory and experience will overcome the limitations of current personal medicine and contribute to the overall world healthcare.

\section{Data Bases}

Elsevier Science: http://www.sciencedirect.com/ CNKI: http://www.cnki.net/ Traditional Chinese Medicine | Syndrome | Pattern | Zheng | Systems Biology | Genomics | Transcriptomics | Proteomics | Metabolomics

\section{Conflict of Interests}

The authors declared that they have no conflicts of interest to this work. And they did not have a direct financial relation with the commercial identity mentioned in this paper.

\section{Authors' Contribution}

J. Dai, J. Fang, S. Sun, and Q. Chen contributed equally to this work and should be considered co-first authors.

\section{Acknowledgments}

This work was supported by the Shanghai Interdisciplinary Cultivation Platform of Outstanding and Innovative Postgraduates, National Science and Technology Major Project (2012ZX10005001-004), and Shanghai “085" Science and Technology Innovation Supporting Project for Top-Grade Discipline Construction.

\section{References}

[1] T. A. Clayton, J. C. Lindon, O. Cloarec et al., "Pharmacometabonomic phenotyping and personalized drug treatment," Nature, vol. 440, no. 7087, pp. 1073-1077, 2006.

[2] Huangdi's Internal Classic.

[3] J. L. Tang, B. Y. Liu, and K. W. Ma, “Traditional Chinese medicine," The Lancet, vol. 372, no. 9654, pp. 1938-1940, 2008.

[4] Y. T. He, A. P. Lu, Y. L. Zha, and I. Tsang, "Differential effect on symptoms treated with traditional Chinese medicine and Western combination therapy in RA patients," Complementary Therapies in Medicine, vol. 16, no. 4, pp. 206-211, 2008.

[5] C. Lu, Q. I. Zha, A. I. Chang, Y. T. He, and A. P. Lu, "Pattern differentiation in traditional chinese medicine can help define specific indications for biomedical therapy in the treatment of rheumatoid arthritis," Journal of Alternative and Complementary Medicine, vol. 15, no. 9, pp. 1021-1025, 2009.
[6] M. A. Hamburg and F. S. Collins, "The path to personalized medicine," The New England Journal of Medicine, vol. 363, no. 4, pp. 301-304, 2011.

[7] J. L. Yuan, H. Zhang, L. Wang et al., "Biochemical characteristics of traditional Chinese medicine syndromes and their elements in patients with hepatitis B cirrhosis," Journal of Chinese Integrative Medicine, vol. 9, no. 4, pp. 374-381, 2011.

[8] Y. Q. Xie, H. Wang, Y. P. Wu, D. H. Yin, Z. S. Wang, and Y. $\mathrm{H}$. Huang, "Association of APOE polymorphisms and insulin resistance with TCM syndromes in type 2 diabetes patients with macroangiopathy," Molecular Medicine Reports, vol. 4, no. 6, pp. 1219-1223, 2011.

[9] X. Q. Yue and Q. Liu, "Analysis of studies on pattern recognition of tongue image in traditional Chinese medicine by computer technology," Journal of Chinese Integrative Medicine, vol. 2, no. 5, pp. 326-329, 2004.

[10] B. Pang, D. Zhang, N. Li, and K. Wang, "Computerized tongue diagnosis based on Bayesian networks," IEEE Transactions on Biomedical Engineering, vol. 51, pp. 1803-1810, 2004.

[11] Y. Wu, Y. Cun, J. Dong et al., "Polymorphisms in PPARD, PPARG and APM1 associated with four types of traditional Chinese medicine constitutions," Journal of Genetics and Genomics, vol. 37, no. 6, pp. 371-379, 2010.

[12] H. M. Ni, Y. P. Wu, and Y. M. He, "Differential expression genes in teenagers with kidney-Yang sthenia constitution by genechiptechnique," Acta Univ Tradit Med Sin Pharm Shanghai, vol. 38, pp. 3-5, 2004 (Chinese).

[13] Y. Feng, Z. H. Wu, X. Z. Zhou, Z. M. Zhou, and W. Y. Fan, "Knowledge discovery in traditional Chinese medicine: state of the art and perspectives," Artificial Intelligence in Medicine, vol. 38, no. 3, pp. 219-236, 2006.

[14] X. C. Liu, H. Liang, Z. Tian, Y. Chen, and L. Zhang, "Comparative proteomic analysis of human kidney-Yang deficiency syndrome serum," Chinese Journal of Biochemistry and Molecular Biology, vol. 23, no. 7, pp. 592-599, 2007 (Chinese).

[15] L. H. Tang, X. D. Wo, D. Z. Lu, M. R. Shi, Y. Li, and L. K. Wo, "Effects of warm and tonify kidney-Yang herbs on liver mitochondria proteome of kidney-Yang deficiency rats," Chinese Pharmaceutical Journal, vol. 42, no. 3, pp. 169-175, 2007.

[16] P. Wang, H. Sun, H. T. Lv et al., "Thyroxine and reserpineinduced changes in metabolic profiles of rat urine and the therapeutic effect of Liu Wei Di Huang Wan detected by UPLCHDMS," Journal of Pharmaceutical and Biomedical Analysis, vol. 53, no. 3, pp. 631-645, 2010.

[17] X. M. Lu, Z. L. Xiong, J. L. Li, S. N. Zheng, T. G. Huo, and F. M. Li, "Metabonomic study on "Kidney-Yang deficiency syndrome" and intervention effects of Rhizoma Drynariae extracts in rats using ultra performance liquid chromatography coupled with mass spectrometry," Talanta, vol. 83, no. 3, pp. 700-708, 2011.

[18] S. Li, Z. Q. Zhang, L. J. Wu, X. G. Zhang, Y. D. Li, and Y. Y. Wang, "Understanding Zheng in traditional Chinese medicine in the context of neuro-endocrine-immune network," IET Systems Biology, vol. 1, no. 1, pp. 51-60, 2007.

[19] T. Ma, C. G. Tan, H. Zhang, M. Q. Wang, W. J. Ding, and S. Li, "Bridging the gap between traditional Chinese medicine and systems biology: the connection of Cold syndrome and NEI network," Molecular BioSystems, vol. 6, no. 4, pp. 613-619, 2010.

[20] S. J. Sun, J. Y. Dai, W. Y. Wang et al., "Metabonomic evaluation of ZHENG differentiation and treatment by Fuzhenghuayu tablet 
in hepatitis-B-caused cirrhosis," Evidence-Based Complementary and Alternative Medicine, vol. 2012, Article ID 453503, 8 pages, 2012.

[21] M. Chen, L. Zhao, and W. Jia, "Metabonomics study on the biochemical profiles of a hydrocortisone-induced animal," Journal of Proteome Research, vol. 4, no. 6, pp. 2391-2396, 2005.

[22] Y. G. Chu, J. Shi, and Y. H. Hu, "Serum proteomic study on hypertension patients with Gan-Dan damp-heat syndrome," Chinese Journal of Integrative Medicine, vol. 30, no. 1, pp. 37-41, 2010.

[23] Z. Z. Guo, S. H. Yu, Y. Guan et al., "Molecular mechanisms of same TCM syndrome for different diseases and different TCM syndrome for same disease in chronic hepatitis B and liver cirrhosis," Evidence-Based Complementary and Alternative Medicine, vol. 2012, Article ID 120350, 9 pages, 2012.

[24] N. J. Zeng, Q. H. Liang, X. G. Xiong et al., "Proteomics analysis and identification on a peripheral blood lymphocyte of Ganyang Huafeng syndrome of cerebral infarction," Chinese Journal of Information on Traditional Chinese Medicine, vol. 15, no. 4, pp. 11-15, 2008 (Chinese).

[25] Q. X. Tan, Z. P. Lu, X. L. Zhong, and X. G. Zhang, "Preliminary study on change of serum proteome in liver stagnation syndrome," Liaoning Journal of Traditional Chinese Medicine, vol. 33, pp. 157-158, 2006 (Chinese).

[26] C. Lu, C. Xiao, L. H. Zhao et al., "Comparison of gene profile of peripheral $\mathrm{CD} 4^{+}$lymphocytes in rheumatoid arthritis with cold and heat syndrome," Chinese Journal of Basic Medicine in Traditional Chinese Medicine, vol. 12, no. 2, pp. 130-133, 2006 (Chinese).

[27] Y. Q. Wang, L. P. Yang, W. J. Ding, H. Gao, and Q. G. Wang, "A report on 15 differential expression genes found in a coldsyndrome genealogy," Journal of Traditional Chinese Medicine, vol. 47, pp. 131-133, 2006.

[28] Y. Gu, C. Lu, Q. L. Cha et al., "Plasma metabonomics study of rheumatoid arthritis and its Chinese medicine subtypes by using liquid chromatography and gas chromatography coupled with mass spectrometry," Molecular Systems Biology, vol. 8, no. 5, pp. 1535-1543, 2012.

[29] C. B. Yang, J. Xue, P. S. Yin, J. S. Zuo, and K. C. Xu, "Expression of blc-2 gene in spleen deficiency syndrome in colorectal carcinoma and the regulatory effect of Jianpikangfu decoction," Academic Journal of First Military Medical University, vol. 25, pp. 1268-1269, 2005 (Chinese).

[30] Z. M. Yang, W. W. Chen, and Y. F. Wang, "Study on gene differential expressions of substance and energy metabolism in chronic superficial gastritis patients of Pi deficiency syndrome and of Pi-Wei hygroyrexia syndrome," Chinese Journal of Integrative Medicine, vol. 32, no. 9, pp. 1180-1187, 2012.

[31] P. Liu, Y. Y. Zhang, and J. Qiao, "Establishment and analysis of serum two-dimensional gel electrophoresis profiles of myasthenia gravis patients with spleen and kidney deficiency syndrome," Journal of Chinese Integrative Medicine, vol. 5, no. 2, pp. 150-154, 2007.

[32] L. Weng, J. Du, W. T. He, and C. Q. Ling, "Characteristic genomics of peripheral blood mononuclear cells of hepatocellular carcinoma patients with liver-kidney yin deficiency syndrome," Chinese Journal of Integrative Medicine, vol. 10, no. 4, pp. 406-415, 2012.

[33] H. S. Zheng, J. Jiang, W. Jia et al., "Research on metabolomics in chronic heart failure with kidney-Yang deficiency," China Journal of Traditional Chinese Medicine and Pharmacy, vol. 25, no. 2, pp. 198-201, 2010 (Chinese).
[34] Y. Guan, H. Zhang, W. Zhang, and S. B. Su, "Analysis of differential gene expression profile in peripheral blood of patients with chronic hepatitis B and syndromes of dual deficiency of liver and kidney yin and accumulation of dampness heat," Chinese Journal of Integrative Medicine, vol. 10, no. 7, pp. 751-756, 2012.

[35] Y. Q. Wang, F. F. Li, W. J. Wang, L. Y. Zhao, L. Guo, and H. F. Wang, "Serum proteomics study of chronic gastritis with dampness syndrome in traditional Chinese medicine," Journal of Chinese Integrative Medicine, vol. 5, no. 5, pp. 514-516, 2007.

[36] Q. Wang, H. Y. Xi, and J. H. Gao, "Study on characteristics of peripheral blood gene expression profile in the obesity with phlegm-dampness constitution," Journal of Traditional Chinese Medicine, vol. 47, pp. 851-858, 2006.

[37] X. J. Ma, H. J. Yin, and K. J. Chen, “Differential gene expression profiles in coronary heart disease patients of blood stasis syndrome in traditional Chinese medicine and clinical role of target gene," Chinese Journal of Integrative Medicine, vol. 15, no. 2, pp. 101-106, 2009.

[38] Z. K. Yuan, X. P. Huang, G. B. Tan et al., "Detective analysis on polymorphism of apolipoprotein $\mathrm{E}$ gene in blood-stasis syndrome of coronary heart disease," Journal of Beijing University of Traditional Chinese Medicine, vol. 31, no. 12, pp. 830-834, 2008 (Chinese).

[39] Z. K. Yuan, L. P. Wang, and X. P. Huang, "The screening and the functional pathway analysis of differential genes correlated with coronary heart disease of blood stasis syndrome," Chinese Journal of Integrative Medicine, vol. 32, no. 10, pp. 1313-1318, 2012.

[40] H. J. Wu, Z. C. Ma, Y. Gao, and S. Q. Wang, "Study on GAP in blood-stasis type of coronary heart disease by using proteomic technique," Chinese Journal of Integrative Medicine on Cardio/Cerebrovascular Disease, vol. 3, pp. 189-191, 2005 (Chinese).

[41] J. Wang, Z. F. Li, H. H. Zhao et al., "Characteristics of urine metabonomics in patients with blood stasis syndrome of $\mathrm{CHN}$ unstable angina," Journal of Beijing University of Traditional Chinese Medicine, vol. 35, no. 4, pp. 284-288, 2012.

[42] Q. Y. Li, Z. Z. Guo, J. Liang et al., "Interleukin-10 genotype correlated to deficiency syndrome in hepatitis B cirrhosis," Evidence-Based Complementary and Alternative Medicine, vol. 2012, Article ID 298925, 6 pages, 2012.

[43] Y. N. Song, H. Zhang, Y. Guan et al., "Classification of traditional Chinese medicine syndromes in patients with chronic hepatitis B by SELDI-based proteinchip analysis," Based Complementary and Alternative Medicine, vol. 2012, Article ID 626320, 10 pages, 2012.

[44] Q. W. Chen, X. Q. Huang, G. J. Yang et al., "Preliminary study of serum metabonomics on Yang deficiency syndrome of patients with primary liver cancer," Chin Arch Tradit Chin Med, vol. 30, no. 3, pp. 526-529, 2012.

[45] T. Wu, M. Yang, H. F. Wei, S. H. He, S. C. Wang, and G. Ji, "Application of metabolomics in traditional Chinese medicine differentiation of deficiency and excess syndromes in patients with diabetes mellitus," Evidence-Based Complementary and Alternative Medicine, vol. 2012, Article ID 968083, 11 pages, 2012.

[46] C. H. Yang, J. M. Lin, and J. Xie, "Study on the metabolic difference of hypertension patients of Gan-Yang hyperactivity syndrome and Yin-Yang deficiency syndrome," Chinese Journal of Integrative Medicine, vol. 32, no. 9, pp. 1204-1207, 2012.

[47] L. Weng, J. Du, W. T. He, and C. Q. Ling, "Characteristic genomics of peripheral blood monouclear cells of hepatocellular carcinoma patients with liver-kidney yin deficiency 
syndrome," Chinese Journal of Integrative Medicine, vol. 10, no. 4, pp. 406-415, 2012.

[48] H. Q. Jiang, Y. L. Li, and J. Xie, "Urine metabonomic study on hypertension patients of ascendant hyperactivity of Gan Yang syndrome by high performance liquid chromatography coupled with time of flight mass spectrometry," Chinese Journal of Integrative Medicine, vol. 32, no. 3, pp. 333-337, 2012.

[49] J. J. Hu, Z. Q. Chen, G. W. Zhong, W. Li, and T. H. Yin, "Comparative proteomics analysis on splenic lymphocytes in migraine rat model with hyperactivity of liver-yang," Chinese Journal of Information on Traditional Chinese Medicine, vol. 15, pp. 34-37, 2008 (Chinese).

[50] G. W. Zhong, J. Hu, Z. Chen et al., "Effect of the formulae for calming the liver and suppressing YANG on lymphocyte proteome in migraine rats with syndrome of hyperactivity of liver-YANG," Journal of Central South University (Medical Sciences), vol. 35, no. 1, pp. 70-76, 2010 (Chinese).

[51] L. Y. Zhou, Z. Q. Chen, and W. Li, "Observation of differential protein of hypothalamus in the hyperthyroidlsm rats with hyperactivity of liver-yang," Chinese Journal of Information on Traditional Chinese Medicine, vol. 14, pp. 18-20, 2007.

[52] X. Zhang, X. Zeng, X. J. Duan, and R. M. Ou, "Reproduction of a rat model of liver-yang hyperactivity syndrome with essential hypertension and analysis of protein expression in liver," Sichuan Journal of Traditional Chinese Medicine, vol. 26, pp. 9-11, 2008.

[53] X. L. Zhong, Z. P. Lu, L. J. Qian, X. H. Liu, Q. X. Tan, and X. G. Zhang, "Differential expression of serum proteome on stagnation on liver-Qi syndrome model rats," China Journal of Traditional Chinese Medicine and Pharmacy, vol. 21, no. 5, pp. 399-401, 2006.

[54] S. Wang, S. J. Zou, X. Y. Chen, and W. J. Gang, "Primary study on changes of gene expression profile in hippocampl of reserpine animal models with spleen-asthenia," Mod J Integr Tradit Chin West Med, vol. 13, pp. 841-844, 2004.

[55] H. G. Luo, J. Ding, G. X. Yue, and J. X. Chen, "Metabonomic study of syndrome of liver Qi stagnation and spleen deficiency in rats," Journal of Chinese Integrative Medicine, vol. 5, pp. 307313, 2007.

[56] Z. Y. Shen, Y. Chen, J. H. Huang, and Z. W. Hu, "The gene expression profile in hypothalamus-pituitary-adrenalthymus(HPAT) axis from EF-treated old rats," Chinese Journal of Immunology, vol. 20, pp. 59-62, 2004 (Chinese).

[57] M. J. Chen, L. P. Zhao, and W. Jia, "Metabonomic study on the biochemical profiles of a hydrocortisone-induced animal model," Journal of Proteome Research, vol. 4, no. 6, pp. 23912396, 2005.

[58] J. X. Liu, L. Miao, X. Z. Li, and Y. H. Pan, "Proteomics research of serum of Chinese experimental mini-pig model with syndrome of phlegm and blood stasis of coronary," Pharmacology and Clinics of Chinese Materia Medica, vol. 26, pp. 73-76, 2010.

[59] L. Tong, S. H. Chen, Z. C. Ma, J. Huang, Y. Ding, and S. Q. Wang, "Accessing gene expression profile of blood deficiency mice using hematopoietic-ralated cytokine oligonucleotide microarrays," Chinese Traditional and Herbal Drugs, vol. 34, no. 7, pp. 625-629, 2003 (Chinese).

[60] W. X. Jian, Z. K. Yuan, and X. P. Huang, "Detection and analysis on plasma metabolomics in patient with coronary heart disease of xin-blood stasis syndrome pattern," Chinese Journal of Integrative Medicine, vol. 30, pp. 579-584, 2010.

[61] Y. Wang, Z. F. Li, J. X. Chen et al., "Study of mini-pig serum of coronary heart disease (chronic myocardial ischemia) with syndrome of blood stasis based on nuclear," Chinese Journal of Analytical Chemistry, vol. 39, no. 8, pp. 1274-1278, 2011 (Chinese).

[62] Z. Q. Pan, Z. Q. Fang, W. L. Lu et al., "Difference of gene expression in adrenal gland of H22 tumor mice of Yang-Qi deficiency syndrome and Qi-Yin-Yang deficiency syndrome," Acta Univ Tradit Med Sin Pharm Shanghai, vol. 22, no. 3, pp. 45-50, 2008 (Chinese).

[63] C. Lu, C. Xiao, G. Chen et al., "Cold and heat pattern of rheumatoid arthritis in traditional Chinese medicine: distinct molecular signatures indentified by microarray expression profiles in CD4-positive T cell," Rheumatology International, vol. 32, no. 1, pp. 61-68, 2012.

[64] Y. Liu, P. Liu, R. Dai et al., "Analysis of plasma proteome from cases of the different traditional Chinese medicine syndromes in patients with chronic hepatitis B," Journal of Pharmaceutical and Biomedical Analysis, vol. 59, pp. 173-178, 2012.

[65] C. L. Lu, X. Y. Qu, and J. G. Jiang, "Proteomics and syndrome of Chinese medicine," Journal of Cellular and Molecular Medicine, vol. 14, no. 12, pp. 2721-2728, 2010.

[66] H. van Wietmarschen, K. Yuan, C. Lu et al., "Systems biology guided by Chinese medicine reveals new markers for sub-typing rheumatoid arthritis patients," Journal of Clinical Rheumatology, vol. 15, no. 7, pp. 330-337, 2009.

[67] P. Sun, G. A. Luo, M. Q. Qiao et al., "Studies on the metabonomics of premenstrual syndrome liver-Qi invasion patient interfered by Jingqianping granules," World Science and Technology/Modernization of Traditional Chinese Medicine and Materia Medica, vol. 12, no. 2, pp. 195-201, 2010.

[68] Y. Qiu, M. Su, Y. Liu et al., "Application of ethyl chloroformate derivatization for gas chromatography-mass spectrometry based metabonomic profiling," Analytica Chimica Acta, vol. 583, no. 2, pp. 277-283, 2007.

[69] Chinese Society of Hepatology and Chinese Medical Association, "The guidelines of prevention and treatment for chronic hepatitis B," Chinese Journal of Hepatology, vol. 13, pp. 881-891, 2005 (Chinese).

[70] Y. X. Zhang and B. H. Wei, "Evaluation criteria of the clinical diagnosis, drug efficacy and TCM syndrome differentiation for cirrhosis (pilot program)," Chinese Journal of Integrative Medicine, vol. 14, pp. 237-238, 1994 (Chinese).

[71] J. F. Boulicaut, F. Esposito, F. Giannotti, and D. Pedreschi, Eds., Proceedings of the 8th European Conference on Principles and Practice of Knowledge Discovery in Databases, Springer, Berlin, Germany, 2004.

[72] S. Li, "Computational Systems Biology-based TCM Research: a case study of cold/heat zheng and associated formula," World Science and Technology/Modernization of Traditional Chinese Medicine and Materia Medica, vol. 9, no. 1, pp. 105-111, 2007.

[73] H. L. Wu, X. M. Ruan, and W. J. Luo, "Cluster analysis on TCM syndromes in 319 coronary artery disease patients for establishment of syndrome diagnostic figure," Chinese Journal of Integrative Medicine, vol. 27, pp. 616-618, 2007.

[74] C. T. Shen, H. Q. Zhang, and X. H. Zhu, "Investigation and analysis on diagnostic standard for TCM syndrome differentiation in 400 patients with climacteric syndrome," Chinese Journal of Integrative Medicine, vol. 24, pp. 517-520, 2004.

[75] W. H. Liu, X. Z. Yan, L. Zhang, Q. Zhang, L. H. Wang, and F. H. $\mathrm{Yu}$, "Metabonomics study on phlegm and blood stasis evolution of hyperlipidemia and atherosclerosis," Liaoning Journal of Traditional Chinese Medicine, vol. 49, no. 5, pp. 738-741, 2008 (Chinese). 


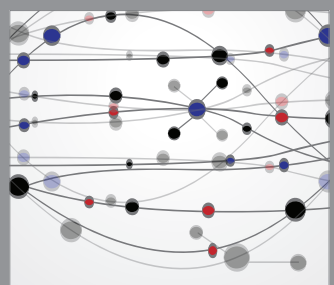

The Scientific World Journal
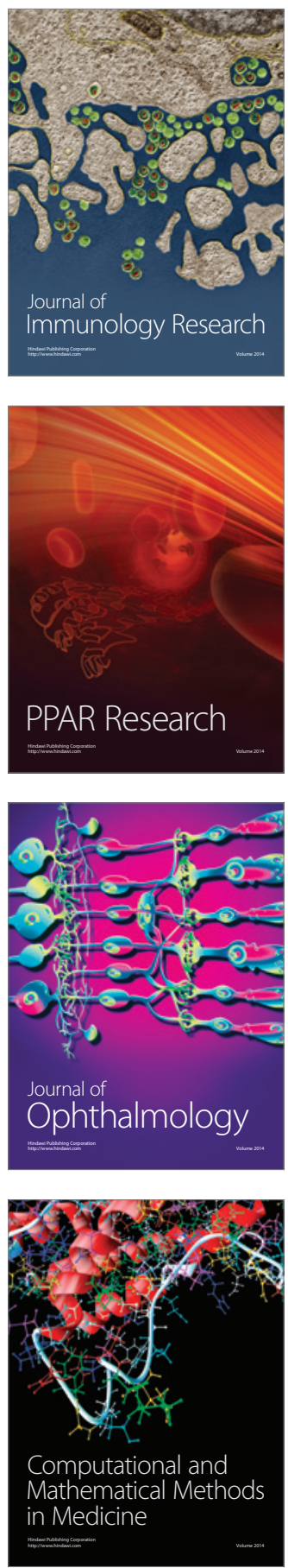

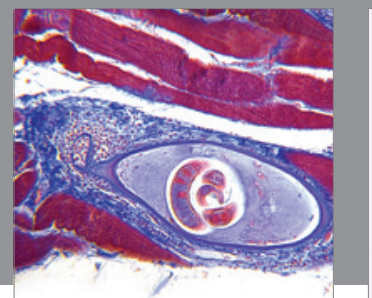

Gastroenterology

Research and Practice
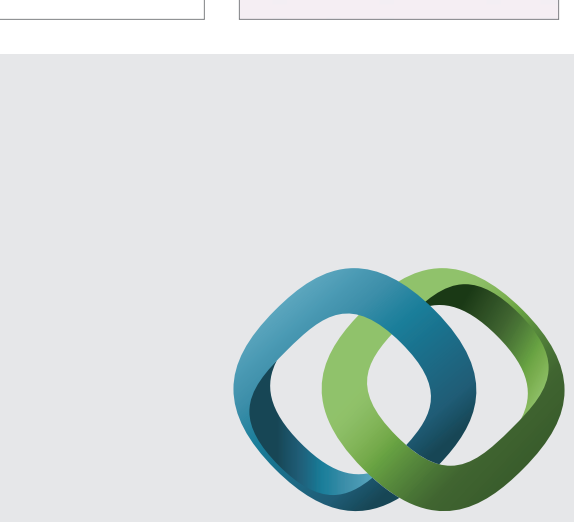

\section{Hindawi}

Submit your manuscripts at

http://www.hindawi.com
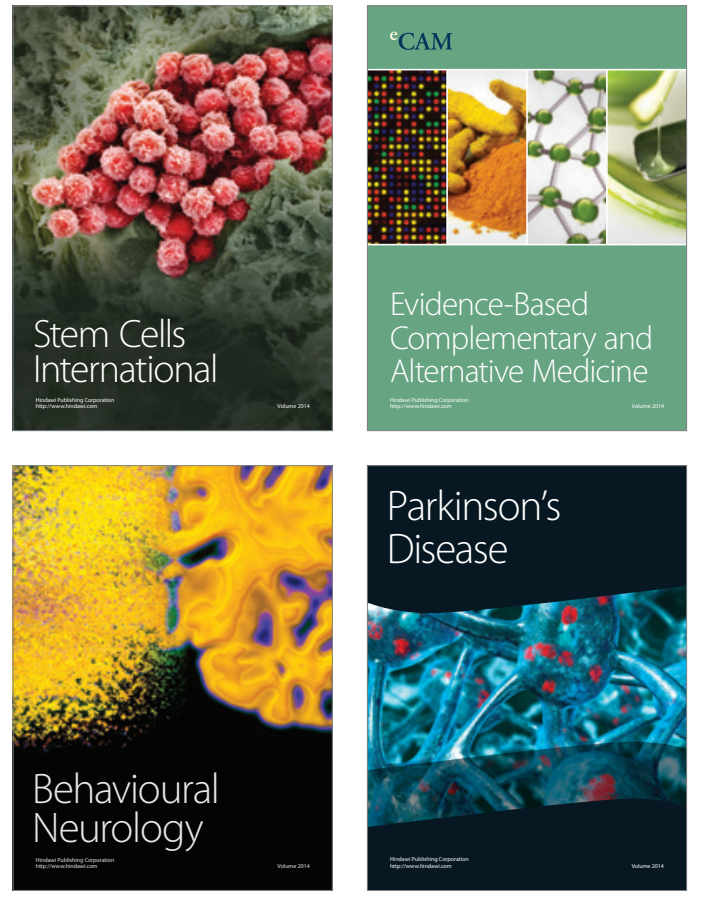
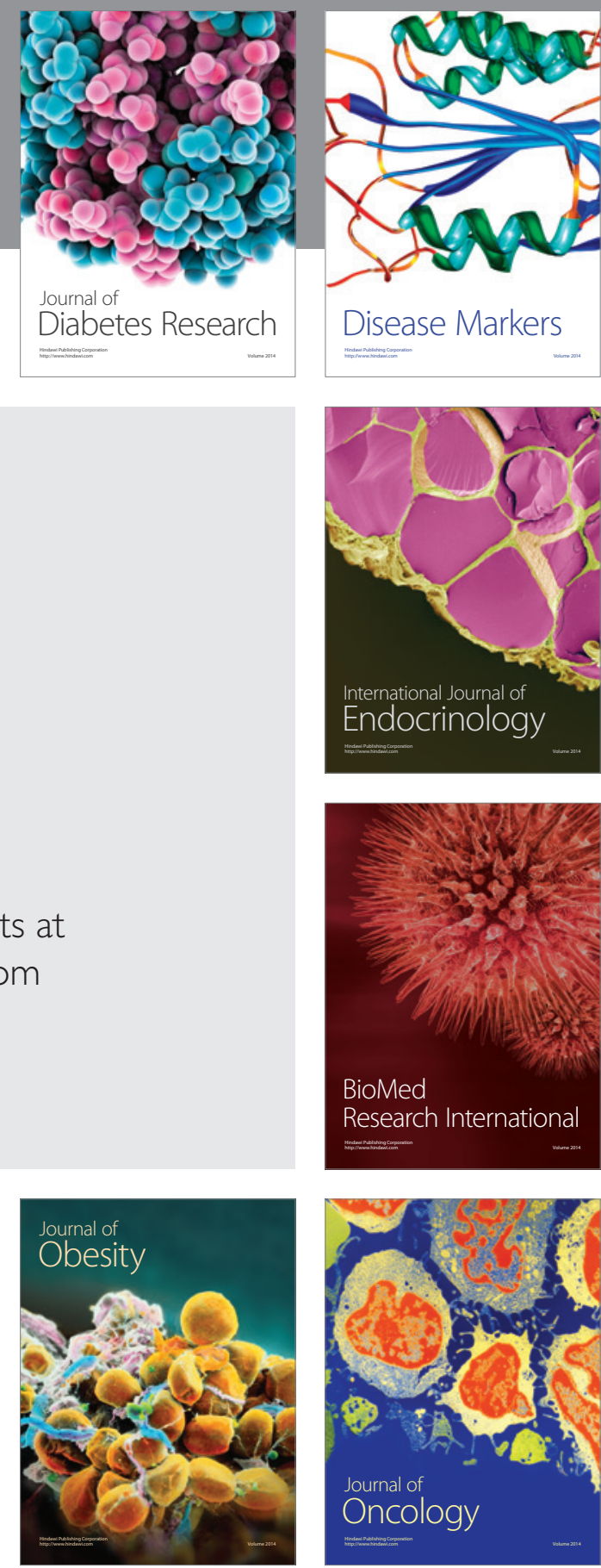

Disease Markers
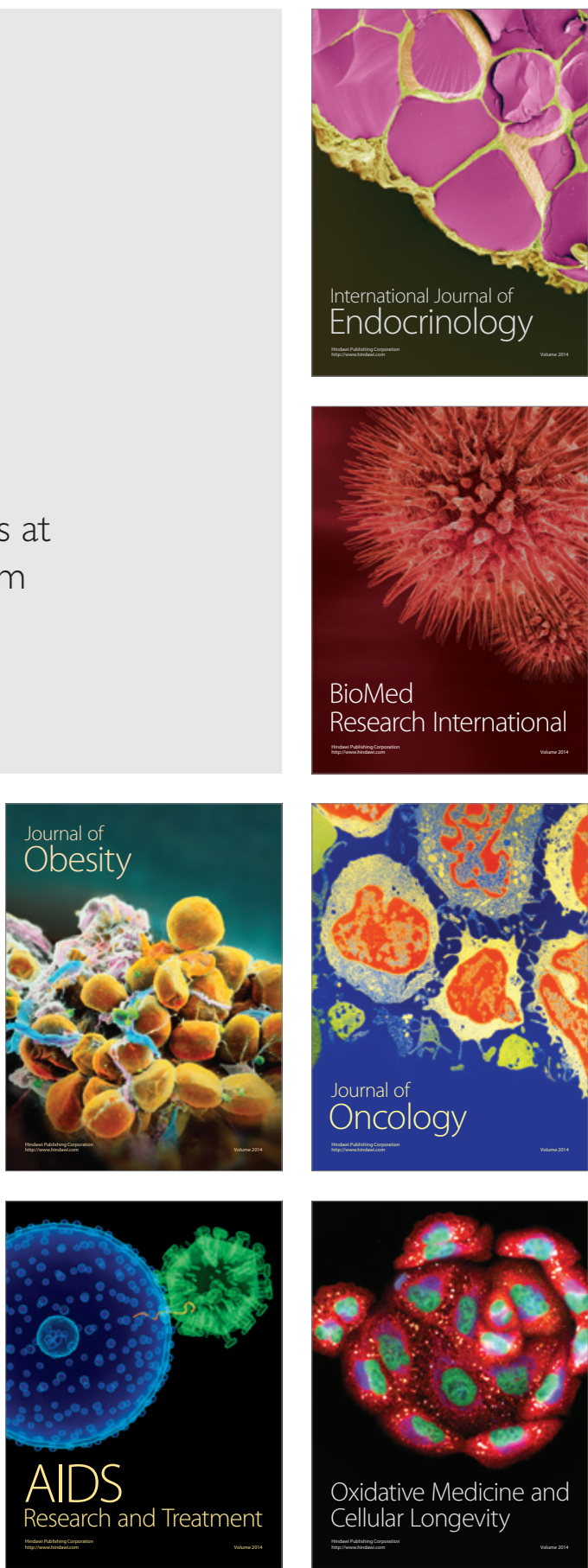